
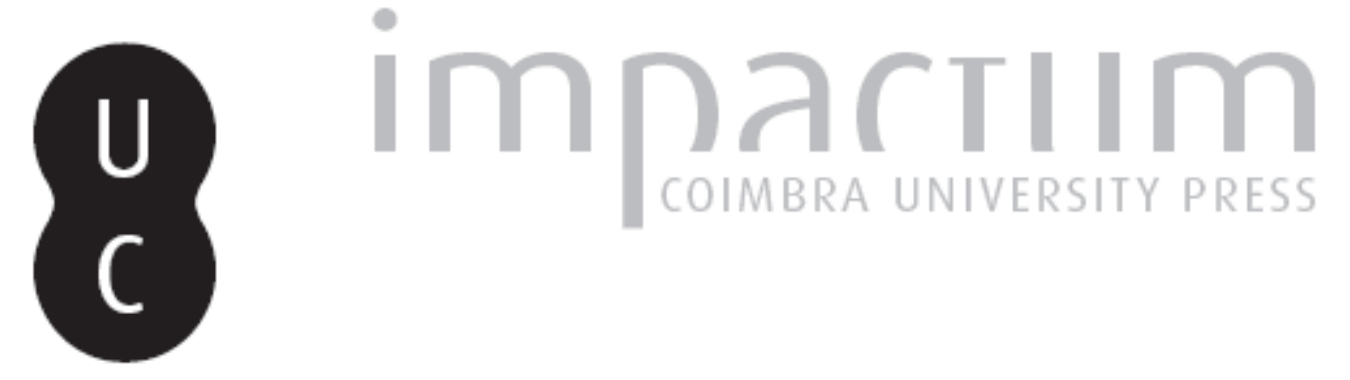

\title{
Aportaciones hídricas en la cabecera fluvial del río Duero: relación con la variabilidad climática y cambios en la cubierta vegetal
}

Autor(es): $\quad$ Ceballos, Antonio; Morán Tejeda, Enrique; Llorente Pinto, José Manuel

Publicado por: Associação Portuguesa de Riscos, Prevenção e Segurança

URL

persistente:

URI:http://hdl.handle.net/10316.2/36194

DOI:

DOI:http://dx.doi.org/10.14195/1647-7723_15_

Accessed : $\quad$ 26-Apr-2023 11:39:24

A navegação consulta e descarregamento dos títulos inseridos nas Bibliotecas Digitais UC Digitalis, UC Pombalina e UC Impactum, pressupõem a aceitação plena e sem reservas dos Termos e Condições de Uso destas Bibliotecas Digitais, disponíveis em https://digitalis.uc.pt/pt-pt/termos.

Conforme exposto nos referidos Termos e Condições de Uso, o descarregamento de títulos de acesso restrito requer uma licença válida de autorização devendo o utilizador aceder ao(s) documento(s) a partir de um endereço de IP da instituição detentora da supramencionada licença.

Ao utilizador é apenas permitido o descarregamento para uso pessoal, pelo que o emprego do(s) título(s) descarregado(s) para outro fim, designadamente comercial, carece de autorização do respetivo autor ou editor da obra.

Na medida em que todas as obras da UC Digitalis se encontram protegidas pelo Código do Direito de Autor e Direitos Conexos e demais legislação aplicável, toda a cópia, parcial ou total, deste documento, nos casos em que é legalmente admitida, deverá conter ou fazer-se acompanhar por este aviso.

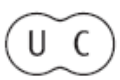




\section{territorium}

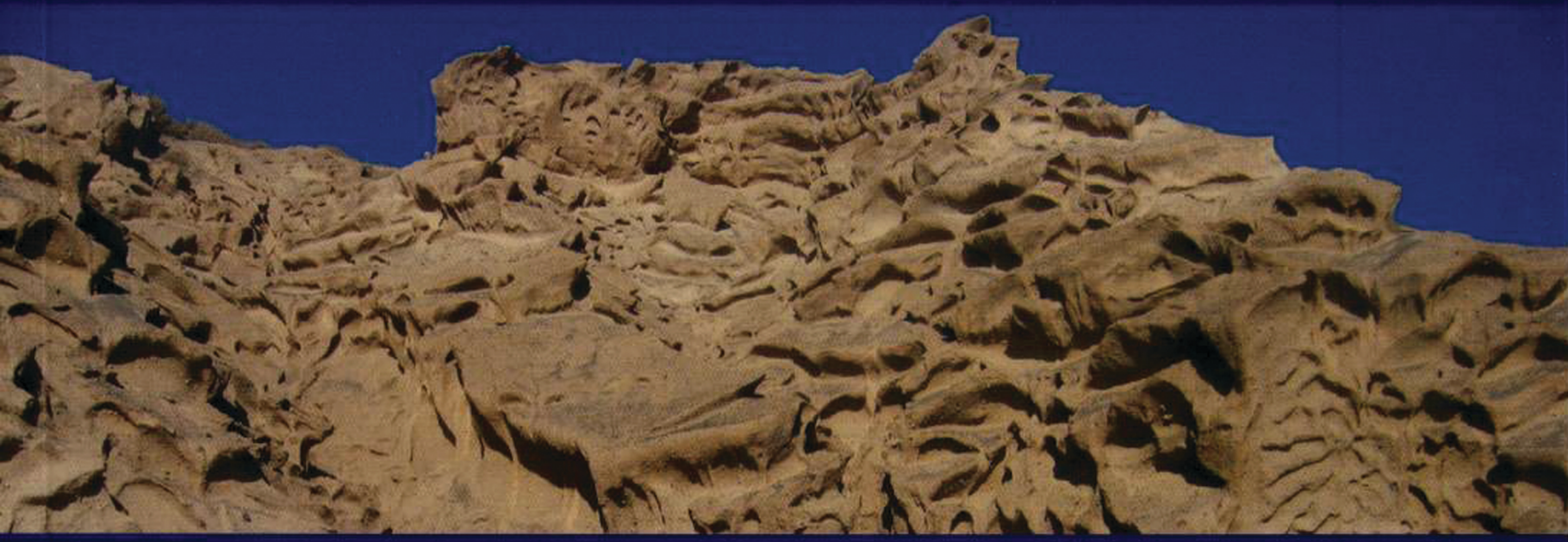

15

Revista da Associação Portuguesa de Riscos, Prevenção e Segurança 2008 


\title{
APORTACIONES HÍDRICAS EN LA CABECERA FLUVIAL DEL RÍO DUERO: RELACIÓN CON LA VARIABILIDAD CLIMÁTICA Y CAMBIOS EN LA CUBIERTA VEGETAL
}

\author{
Antonio Ceballos, Enrique Morán Tejeda \& José Manuel Llorente Pinto \\ Departamento de Geografía. Universidad de Salamanca (España)
}

\begin{abstract}
RESUMEN
En este trabajo se analizan los cambios ocurridos en la evolución temporal de los caudales y del régimen hídrico en la cabecera fluvial del río Duero y su relación con la tendencia de las temperaturas, las precipitaciones y la superficie ocupada por el bosque. Los resultados muestran un decrecimiento significativo en las aportaciones hídricas anuales debido a la marcada reducción de las aportaciones entre los meses de febrero y septiembre, con un aumento sustancial del número de días al año con un caudal inferior a la tercera parte del caudal diario medio anual ("régimen de aguas bajas"). La buena correlación entre precipitaciones y aportaciones anuales ( $R$ $=0,72$, valor-p $<0,001$ ) y la ausencia de tendencia alguna en el análisis de los residuales prueban que el moderado avance de la superficie forestal durante las últimas décadas de momento no es suficiente para influir de manera determinante en la reducción de las aportaciones registrada. No obstante, la falta de correspondencia entre las tendencias mensuales de precipitaciones y aportaciones y la destacable subida de las temperaturas sugieren un aumento del consumo de agua por parte del bosque que muy probablemente afecte de forma claramente perceptible a las aportaciones anuales de la cuenca a corto-medio plazo. Finalmente, la disminución de las precipitaciones y los cambios ocurridos en su reparto intraanual han afectado al régimen del río, que ha pasado de tener un máximo invernal de carácter pluvial a un régimen bimodal con un pico principal en invierno y otro secundario en primavera. Esta circunstancia sin duda debe considerarse en la gestión de los embalses situados aguas abajo.
\end{abstract}

Palabras clave: aportaciones hídricas, variabilidad climática, cambios en el bosque, río Duero.

\section{RESUMO}

Neste trabalho analisam-se as mudanças ocorridas na evolução temporal dos caudais e do regime hídrico na cabeceira fluvial do rio Douro e a sua relação com a tendência das temperaturas, precipitações e superfície ocupada pelo bosque. Os resultados mostram um decréscimo significativo nas contribuições hídricas anuais devido à marcada redução das contribuições entre os meses de Fevereiro e Setembro, com um aumento substancial do número de dias por ano com caudal inferior à terça parte do caudal diário médio anual ("regime de águas baixas"). A boa correlação entre precipitações e contribuições anuais $(R=0,72$, valor-p $<0,001)$ e a ausência de qualquer tendência na análise dos resíduos provam que o avanço moderado da superfície florestal durante as últimas décadas de momento não é suficiente para influir de maneira determinante na redução de aportes registada. Não obstante, a falta de correspondência entre as tendências mensais de precipitações e contribuições e a destacável subida das temperaturas sugerem um aumento do consumo de água por parte do bosque que muito provavelmente afecte de forma claramente perceptível as contribuições anuais da bacia a curto-médio prazo. Finalmente, a diminuição das precipitações e as mudanças ocorridas na sua repartição intra-anual afectaram o regime do rio, que passou de um máximo invernal da carácter pluvial a um regime bimodal com um pico principal no Inverno e outro secundário na Primavera. Esta circunstância deve, sem dúvida, considerar-se na gestão das albufeiras situadas a jusante.

Palavras chave: contribuições hídricas, variabilidade climática, mudanças no bosque, rio Douro. 


\begin{abstract}
This work analyses the changes in the evolution of river discharges and river regime in the Duero river headwaters over the past 5 decades. It further explores their relation with the tendencies observed for temperatures, precipitation, and forest cover extension. Results show significant losses on annual runoff, due to the notable decrease in river discharges during February - September. Moreover, the number of days with a flow lower than the third of the "mean annual daily flow" has clearly decreased. Linear regression developed shows a good correlation between annual precipitation and annual runoff $(R=0.72$, $p$-value $<0.001)$ and the plot of residuals don't show any significant tendency. These facts suggest that the forest growth undergone in the last few decades hasn't had a clear influence on the reduction of river discharges. Nevertheless the absence of correlation between the monthly tendencies for precipitation and runoff, and the important increase in temperatures, indicate an increase in the volume of water consumed by the forest that will probably be perceptible in the evolution of runoff in a short/ middle term basis. Finally, the decrease in precipitation and the changes occurred in its monthly distribution have clearly affected the river regime. This has changed from having a maximum peak in winter during the first 2 decades of the studied period to a bimodal regime in the last 2 decades, with a principal peak in winter and a secondary peak in spring. This fact has to be considered by water administration to develop a responsible management in dams situated downstream.
\end{abstract}

Key words: water discharge, climatic variability, forest change, Douro river.

\title{
RÉSUMÉ
}

Dans ce travail nous analysons les changements arrivés dans l'évolution temporelle des débits et du régime hydrique dans le bassin versant du fleuve Duero et son rapport avec la tendance des températures, des précipitations et de la superficie occupée par le bois. Les résultats montrent une décroissance significative des eaux d'alimentation annuels à cause d'une réduction remarquable des apports entre févriers et mars, et une augmentation importante du nombre de jours par an où le débit est inférieur au tiers du débit journalier moyen annuel ("régime de basses eaux ou étiage »). La bonne corrélation entre précipitations et apports annuels $(R=0.72$, valeur-p $<0.001)$ et l'absence de toute tendance dans l'analyse des résiduelles montrent que la croissance modérée de la superficie forestière pendant les dernières décennies n'est pas suffisante, pour le moment, pour influencer d'une manière déterminante dans la réduction constatée des apports. Cependant, l'absence de corrélation entre tendances mensuelles des précipitations et apports et la hausse remarquable des températures suggèrent une augmentation de la consommation d'eau de la part du bois, qui touchera probablement d'une façon clairement perceptible les apports annuels du bassin dans un délai court-moyen. Finalement, la diminution des précipitations et les mutations arrivées dans sa répartition interannuelle ont touché le régime du fleuve, qui est passé d'un maximum hivernal à caractère pluvial à un régime bimodal avec un pic principal en hiver et un autre secondaire au printemps. Cette circonstance doit sans doute être retenue pour une gestion des barrages situés en aval.

Mots clé: apports hydriques, variabilité climatique, changements dans la forêt, fleuve Duero. 


\section{Introducción}

El déficit de lluvias sufrido durante los años 2004, 2005 y 2007 ha reabierto el debate social sobre la disponibilidad de los recursos hídricos en la Península Ibérica. El almacenamiento de agua en los embalses depende directamente de las escorrentías generadas en las cuencas de cabecera, a su vez determinadas por la evolución de variables climáticas como temperaturas y precipitaciones, así como por la dinámica de la propia cobertura del suelo.

La evolución temporal de las temperaturas en España durante los últimos 150 años ha mostrado un incremento generalizado de $0,1^{\circ} \mathrm{C}$ por década (BRUNET et al., 2007), mientras que en el caso de las precipitaciones no se ha observado una tendencia clara extrapolable al conjunto del territorio (FONT TuLLot, 2000). Uno de las cambios ambientales más notables que ha experimentado el continente europeo en las últimas décadas, y la Península Ibérica no representa excepción alguna, ha sido el abandono de las actividades en el medio rural, debido al éxodo poblacional, y el consecuente avance del bosque, con las implicaciones que ello conlleva respecto a la reducción de escorrentías superficiales (Brown et al, 2005; VERBURG et al., 2006).

En relación con la evolución temporal de las aportaciones de los ríos, en España sólo existen estudios de diagnóstico regional, con una especial incidencia en la cuenca del Ebro. En este sentido, el trabajo de Beguería et al. (2003), realizado a partir de los datos pertenecientes a una red de cuencas de cabecera en la cuenca hidrográfica del Ebro, relacionó el efecto de las oscilaciones climáticas y de los cambios de uso del suelo en los caudales, durante el período 1945-1995, observando una reducción de los mismos, estimada en un $30 \%$, como consecuencia de la recuperación de las masas forestales. Estos resultados fueron posteriormente confirmados por GALLART \& LLORENS (2004), dentro de la misma unidad de estudio, pero analizando cuencas diferentes, señalando una tendencia decreciente en la aportación de estos ríos (disminución anual de un $0,68 \%$ del caudal medio), más relacionada con cambios en el uso del suelo que con cambios registrados en las precipitaciones y evapotranspiración potencial como consecuencia de un aumento de las temperaturas. Sin embargo estos resultados contrastan en parte con los publicados por el Ministerio de Medio Ambiente (MIMAM, 2000) para el conjunto de la cuenca del Ebro. Según este documento, el caudal medio del río Ebro en su desembocadura ha decrecido en un $40 \%$ en los últimos 50 años como consecuencia de una disminución de las precipitaciones y del incremento de la demanda de agua para riego.

En relación con la cuenca del Duero existen estudios realizados en pequeñas cuencas como los de MACHíN et al (2005) en el río Almarza (España), Ceballos \& Morán Tejeda (2006) en la cabecera del Tormes (España), Nunes (2007) en el río Côa (Portugal) o Ceballos et al. (2008) en las cuencas del Tormes, Huebra y Águeda que apuntan una reducción de los caudales relacionada con un descenso de las precipitaciones, pero sin concretar el papel real del avance del bosque sobre los cambios observados en las aportaciones hídricas.

El presente estudio tiene como principal objetivo analizar los cambios en la evolución temporal de las aportaciones hídricas, caudales de diversa magnitud y del régimen hídrico en la cabecera fluvial del río Duero y su relación con la tendencia observada en temperaturas, precipitaciones y área de superficie forestal. La discusión de los resultados obtenidos comparándolos con los publicados en estudios previos en la misma red hidrográfica tratará de definir los factores claves para entender la naturaleza de los cambios ante los cuales habrá que estar muy pendientes en las próximas décadas.

\section{2. Área de estudio}

La primera estación de aforos instalada en el cauce del río Duero corresponde a Molinos de Duero, provincia de Soria, y está localizada a 4 15' 18 "N y $2^{\circ} 47^{\prime} 05^{\prime \prime} \mathrm{O}$. Este punto permite delimitar una cuenca de cabecera con una superficie de $135 \mathrm{~km}^{2}$ y una altitud que oscila entre los $2.230 \mathrm{~m}$ en la Sierra de Urbión y los $1.101 \mathrm{~m}$ del propio aforo (Figura 1).

Las litologías predominantes son de origen detrítico con matriz silícea, abundando las alternancias de calcoarenitas con limolitas y de cuarzoarenitas con arcilloitas $y$ en menor medida los conglomerados (IGME, 1986 y 1988).

La precipitación anual promedio de las estaciones meteorológicas ubicadas en la vertiente sur de la Sierra de Urbión oscila entre los $695 \mathrm{~mm}$ del Embalse de la Cuerda del Pozo y los $941 \mathrm{~mm}$ de Vinuesa, con dos meses secos (julio y agosto), aunque con precipitaciones siempre por encima de los $25 \mathrm{~mm}$. La temperatura media anual ronda los $10,5^{\circ} \mathrm{C}$, siendo enero el mes más frío con $3^{\circ} \mathrm{C}$ y julio el más cálido con $20^{\circ}$ C. Debido al efecto combinado de altitud y longitud, el clima característico del espacio 


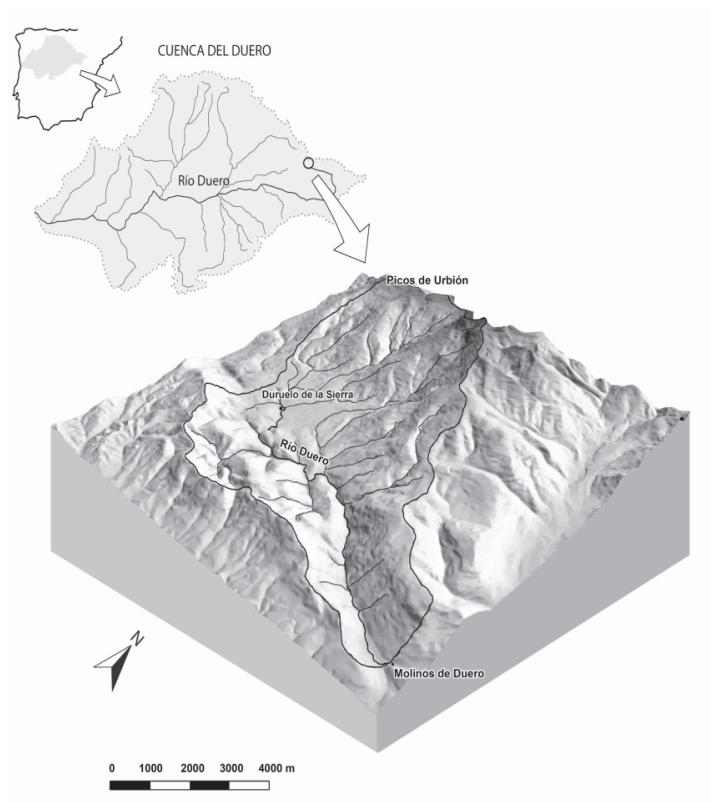

Figura 1: Localización de la zona de estudio y modelo digital del terreno de la cuenca

geográfico estudiado puede definirse como mediterráneo continental.

La cubierta vegetal consiste en una mancha forestal continua y madura de pino albar (Pinus sy/vestris) con intercalaciones de roble melojo (Quercus pyrenaica) en las zonas más bajas y la presencia puntual de hayas (Fagus sylvatica) en los enclaves más húmedos. La cobertura de arbustos (generalmente ericáceas - Erica sp.-, con la presencia de algunas leguminosas de los géneros Genista y Cytisus) y herbáceas se reduce a las zonas más clareadas. Este bosque de pinos ha sido históricamente aprovechado por el hombre con la extracción de madera, lo cual ha repercutido en su mantenimiento mediante repoblaciones forestales $y$ favoreciendo al pino frente a otras especies competidoras (RUIz DE LA TORRE, 1995).

\section{Métodos y técnicas}

Para el estudio de las temperaturas se han utilizado las series mensuales y anual del observatorio meteorológico de la ciudad de Soria (perteneciente al Instituto Nacional de Meteorología, Ministerio de Medio Ambiente) debido a que presenta una situación geográfica comparable y cercana respecto a la zona de estudio (4146'00"N; 2²8'00"O; $1.082 \mathrm{~m}$ ) y ofrece una serie de datos larga y continua en el tiempo (1959-2003, $n=45)$. Para determinar las anomalías térmicas se calculó la diferencia en ${ }^{\circ} \mathrm{C}$ entre la temperatura media correspondiente a cada año y/o mes, y la media del último periodo climático normal
(CLINO 1961-90). El resultado se expresó gráficamente observando la tendencia mediante la representación de la media móvil de 5 años (IPCC, 2001 1). Para constatar el grado de significación de la tendencia (positiva, negativa o nula), con un determinado nivel de confianza, se aplicó un test no parámetrico como la correlación de rangos de Spearman -Rs- (SNeYERS, 1992; YUE et al., 2002).

La única estación pluviométrica ubicada dentro del perímetro de la cuenca de cabecera del Duero es la de Covaleda. Sin embargo su serie de datos es corta (los registros comienzan en 1968) y sobre todo presenta numerosas lagunas. Por este motivo, se ha analizado la serie correspondiente al Embalse de la Cuerda del Pozo situado en las proximidades de Molinos de Duero (4 152' 17"N; 242' 17"O; 1.150 $\mathrm{m})$, ya que ofrece una serie de datos larga y continua (1959-2003, n = 45) y mantiene una correlación altamente significativa con la serie de Covaleda $\left(\mathrm{r}^{2}=\right.$ $0,64$; valor-p $<0,001)$. El análisis consistió en determinar la dispersión de la muestra observando la frecuencia de años secos y húmedos según proponen SCIAN \& DonNARI (1997), para quienes año seco es aquel con valores inferiores a la precipitación media anual menos la desviación estándar (SD) y año húmedo es aquel con un registro superior a la media más la desviación estándar. Para examinar la tendencia temporal de las series mensuales y anual, así como su grado de significación, también se aplicó el test de Spearman. Para definir el grado de variabilidad intra-anual de la precipitación y su evolución temporal a lo largo del período de estudio, se aplicó el índice de concentración de lluvia (Precipitation Concentration Index, PCI) según la propuesta de varios autores (OLIVER, 1980; DE LUIS et al., 2001).

$$
P C I=100 * \frac{\sum_{i=1}^{12} p_{i}^{2}}{\left(\sum_{i=1}^{12} p_{i}\right)^{2}}
$$

donde $p_{i}$ es la cantidad de precipitación de un determinado número de meses $i$. Valores inferiores a 10 indican una distribución uniforme de la precipitación mensual a lo largo del año, valores comprendidos entre 11 y 20 denotan una cierta estacionalidad, y valores superiores a 20 son indicadores de una apreciable variabilidad en el reparto mensual de las precipitaciones anuales.

Respecto al análisis hidrológico, se ha procedido al estudio de la evolución temporal de las aportaciones mensuales y anuales $\left(\mathrm{Hm}^{3}\right)$, caudales medios diarios máximos anuales, caudales máximos instantáneos 
anuales, así como el de las crecidas medidas en la estación de aforos de Molinos de Duero entre los años hidrológicos 1959-60 y 2003-04, aplicando los tests estadísticos mencionados previamente para valorar la tendencia de las series. Se trata de una cuenca de cabecera no afectada por ningún embalse y por tanto representativa del régimen natural del río. En el estudio de la variabilidad de la frecuencia de las crecidas se han considerado los siguientes criterios para definir su magnitud (GARCíA RuIz et al., 2001 ): I) Días de crecidas moderadas o días en que se supera en 3 veces el caudal medio anual ( $Q \times 3)$. II) Días de avenidas o días en que supera en 5 veces el caudal medio anual (Q×5). III) Días de avenidas de baja frecuencia y alta intensidad o días en que se supera en 10 veces el caudal medio anual ( $Q \times 10)$.

Con objeto de comparar, de forma estandarizada, la evolución a lo largo del tiempo de precipitaciones y temperaturas con la observada en las aportaciones hídricas, este conjunto de variables se han normalizado aplicando el índice $F$ propuesto por SHORTHOUSE \& ARNELL (1999) y adoptado por BEGUERÍA et al. (2003) de acuerdo a la siguiente formulación:

$$
F_{i j}=\frac{X_{i j}-A v_{j}}{S_{j}}
$$

donde $F_{i j}$ es el resultado del índice para el año $i$ y el mes $j, X_{i j}$ es el valor mensual del año $i$ y el mes $j, A v_{j}$ es el valor medio mensual del mes i y $S_{i}$ es la desviación estándar de los valores mensuales del mes $j$. El resultado de esta operación es un índice sintético cuyo valor 0 corresponderá a aquellos años con un registro similar a $A v_{i}$ y el valor \pm 1 corresponderá a aquellos años con un registro igual a $A v_{i} \pm S$. Por medio de la representación gráfica de las series temporales normalizadas se podrá apreciar el paralelismo, o no, entre el comportamiento de las variables climáticas y el de las propias aportaciones a través del tiempo.

Los cambios habidos en la cubierta forestal de la cuenca han sido estimados cartografiando la cubierta vegetal mediante la fotointerpretación de las fotografías aéreas correspondientes al vuelo americano de 1957 y de ortofotos tomadas en 2002. Las fotografías aéreas fueron previamente ortorrectificadas para adaptar su geometría a la de las fotos, convirtiendo la perspectiva cónica de la foto en una proyección ortogonal asignando a cada punto de la imagen coordenadas $x, y, z$, mediante un SIG especializado y un modelo digital del terreno.

La relación entre las distintas variables hidroclimáticas ha sido establecida mediante modelos de regresión lineal y múltiple, analizándose también la tendencia temporal de los residuales. El hecho de que los residuales muestren algún tipo de tendencia o salto brusco puede ser interpretado como una señal de la influencia del bosque sobre las aportaciones hídricas.

\section{Resultados}

\subsection{Variables climáticas: temperaturas y precipitaciones}

Considerando la serie de temperaturas del último período climático normal (CLINO 1961-90), la temperatura media anual correspondiente al observatorio de Soria ha sido $10,5^{\circ} \mathrm{C}$, con enero como mes más frío $\left(2,9^{\circ} \mathrm{C}\right)$ y julio como el más cálido $\left(19,9^{\circ} \mathrm{C}\right)$. Durante la totalidad del período analizado (1959-2003) las temperaturas medias anuales han mostrado una evolución positiva con un incremento significativo al $99 \%(R s=0,52)$ de $1,2^{\circ} \mathrm{C}$ en 45 años. No obstante, la figura 2 refleja dos períodos muy contrastados: entre 1959 y 1971 es clara la tendencia negativa de las temperaturas medias anuales respecto al período de referencia (1961-90), mientras que entre 1972 y 2003 el aumento de las temperaturas es muy acusado y significativo: $1,70^{\circ} \mathrm{C}$ en 32 años (Rs $=$ 0,72 ; valor-p $<0,001$ ).

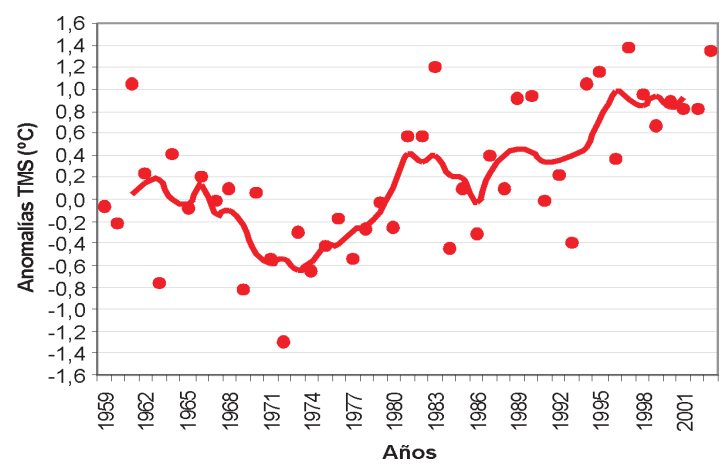

Figura 2: Anomalías térmicas anuales respecto al período de referencia 1961-90 (media móvil de 5 años). Observatorio Meteorológico de Soria, 1959-2003.

La evolución de las anomalías térmicas analizando las series mensuales (figura 3) muestra que durante la totalidad del periodo (1959-2003) solamente en tres meses el incremento de las temperaturas ha sido significativo (marzo, agosto y diciembre), mientras que si consideramos las mismas series a partir de 1972, el número de meses con un calentamiento significativo se eleva a 5, concentrándose en las estaciones primaveral y estival.

La precipitación media anual correspondiente al Embalse de Cuerda del Pozo durante el período 1959 - 

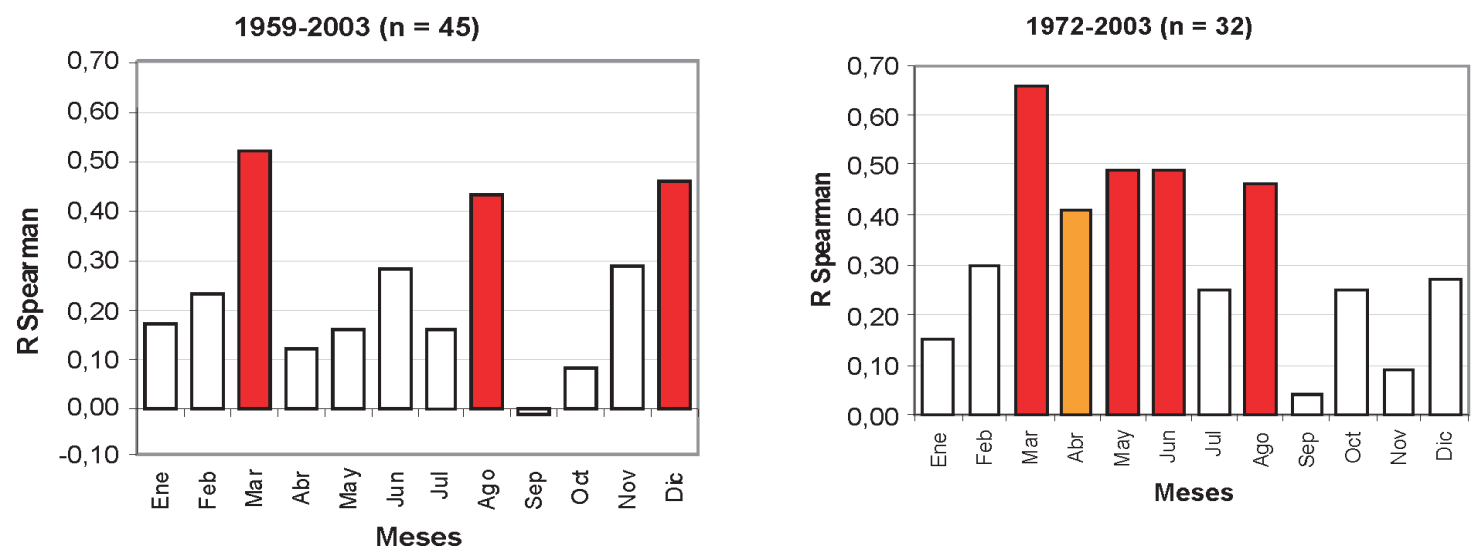

Figura 3: Análisis de las series mensuales de temperaturas correspondientes al Observatorio Meteorológico de Soria, mediante la correlación de rangos de Spearman. Trama blanca: sin correlación. Trama naranja: correlación significativa a un nivel de confianza del 95 \%. Trama roja: correlación significativa a un nivel de confianza del 99 \%. Izquierda: período 1959-2003. Derecha: período 1972-2003.

2003 ha sido de 695 mm, con una desviación estándar igual a $162 \mathrm{~mm}$ y un coeficiente de variación del $23,5 \%$. El ajuste lineal de la serie refleja un descenso estimado en $150 \mathrm{~mm}$ a lo largo del tiempo (figura 4), pero sin ser significativo desde un punto de vista

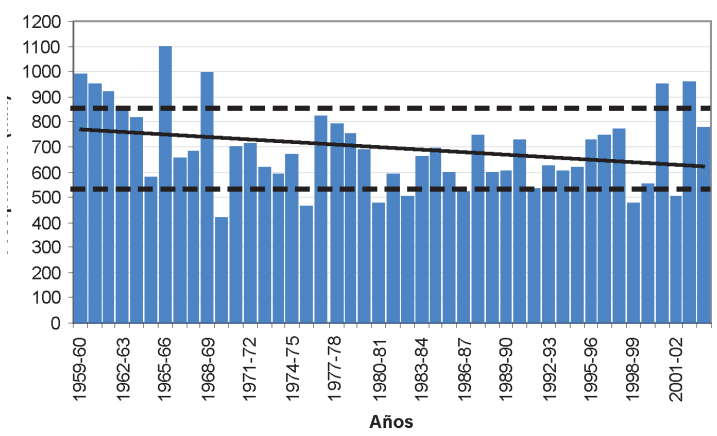

Figura 4: Evolución interanual de las precipitaciones en la estación pluviométrica del Embalse de Cuerda del Pozo, 1959-2003 (líneas discontinuas: precipitación media \pm STD; $695 \pm 162 \mathrm{~mm}$ ).

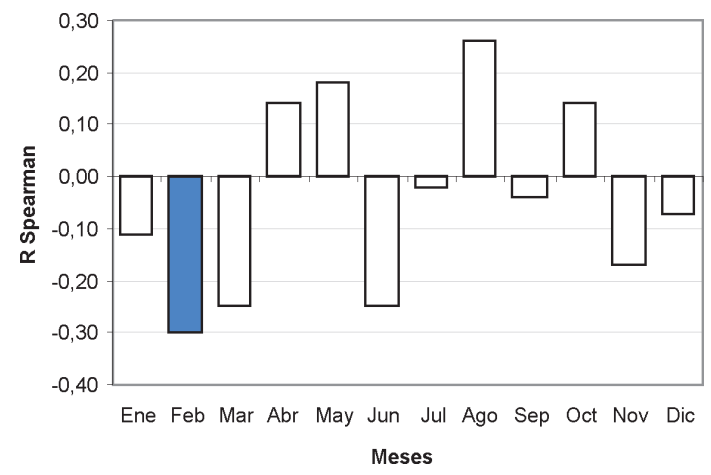

Figura 5: Análisis de las series mensuales de precipitaciones (Observatorio Meteorológico del Embalse de Cuerda del Pozo, 1959-2003) mediante la correlación de rangos de Spearman. Trama blanca: sin correlación. Trama azul: correlación significativa a un nivel de confianza del $95 \%$.

estadístico. Este descenso de las precipitaciones no se ha visto acompañado de un aumento de la variabilidad interanual de las mismas, destacando el número de años con registros que podrían considerarse normales (precipitación comprendida entre la media \pm SD). El análisis de la tendencia temporal de las series mensuales de precipitación (figura 5) muestra el predominio de los meses con

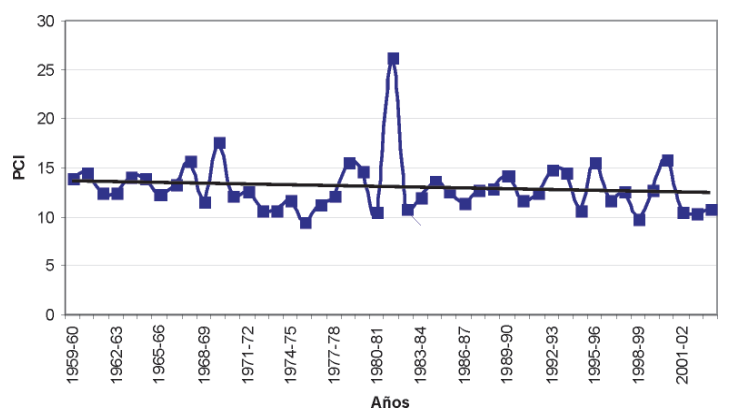

Figura 6: Evolución interanual del $\mathrm{PCl}$ (Precipitation Concentration Index) en la estación pluviométrica del Embalse de Cuerda del Pozo, 1959-2003.

tendencia negativa, concentrados especialmente en las estaciones otoñal e invernal, aunque siendo sólo estadísticamente significativa en febrero.

El análisis de la evolución temporal de la distribución intranual de las precipitaciones, expresada mediante el $\mathrm{PCl}$, no muestra ningún tipo de tendencia estadísticamente significativa, predominando además los valores por debajo de 15, lo cual sugiere un reparto relativamente uniforme de las precipitaciones a lo largo del año (figura 6).

\subsection{Caudales: Aportaciones fluviales y avenidas}

La aportación media anual medida en la estación de aforos de Molinos de Duero durante el período 1959. 60/2003-04 ha sido de 95,5 $\pm 51,7 \mathrm{Hm}^{3}$, con un coeficiente de variación igual a $54 \%$. El análisis de la 
serie interanual de los totales anuales de dicha variable (figura 7) muestra una tendencia negativa en el tiempo $(R s=-0,39)$, significativa al $99 \%$, y que mediante un ajuste lineal podemos estimar en un 59 $\%$. El análisis de la tendencia temporal de las series mensuales de aportaciones muestra un descenso muy marcado y continuo entre febrero y septiembre, siendo especialmente significativo en marzo, junio, julio, agosto y septiembre (figura 8 ).

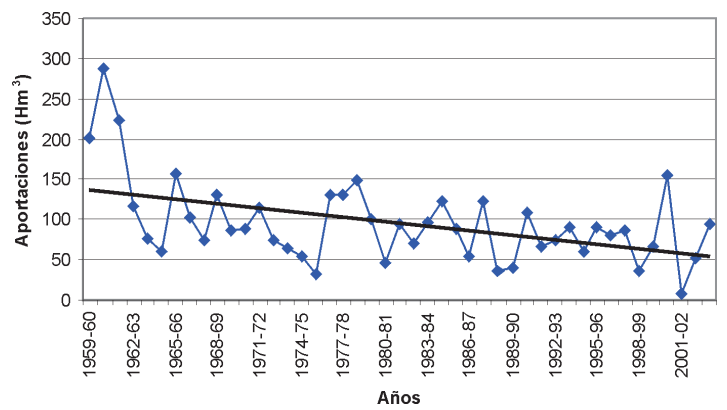

Figura 7: Evolución interanual de las aportaciones en la estación de aforos de Molinos de Duero, 1959-60/2003-04.

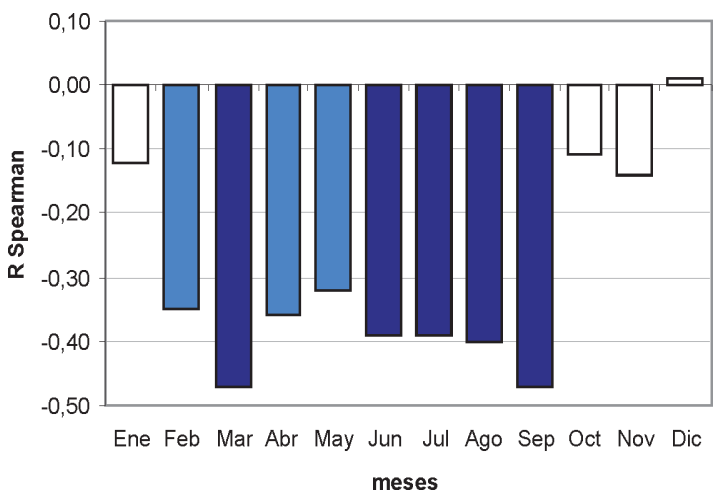

Figura 8: Análisis de las series mensuales de aportaciones (Estación de aforos de Molinos de Duero, 1959-2003) mediante la correlación de rangos de Spearman. Trama blanca: sin correlación. Trama azul claro: correlación significativa a un nivel de confianza del $95 \%$. Trama azul oscuro: correlación significativa a un nivel de confianza del $99 \%$.

Considerando la totalidad de la serie de datos, la distribución mensual de las aportaciones es la propia de un régimen pluvial, con un pico claro en el mes de enero tras la acumulación de lluvia en noviembre, diciembre y enero (figura 9). No obstante, si comparamos los datos medios correspondientes a las dos primeras décadas de la serie con los de las dos últimas (figura 10), el máximo invernal de carácter pluvial era muy claro en la primera mitad de la serie, mientras que el apreciable descenso de las precipitaciones en enero, febrero y marzo, y el leve ascenso en abril, han derivado en la modificación del régimen fluvial, que pasa a tener un carácter bimodal, con un pico principal invernal y uno secundario en primavera.

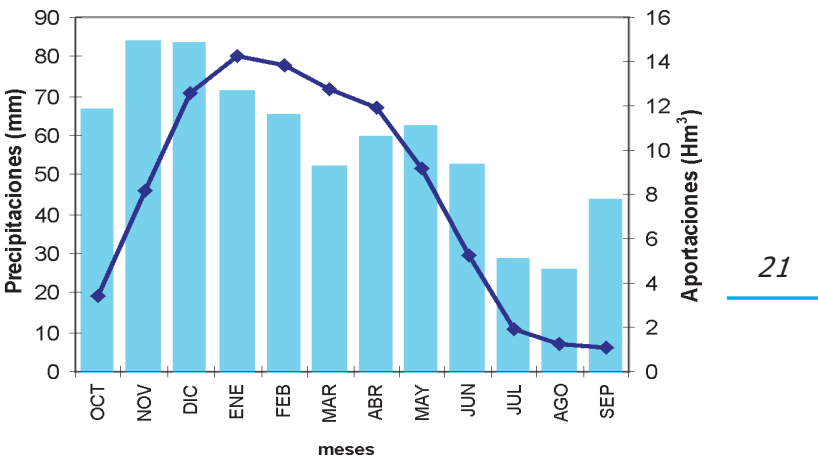

Figura 9: Distribución mensual de las aportaciones (línea) y precipitaciones (barras) en la cabecera del río Duero (195960/2003-04)

Junto al análisis de las aportaciones fluviales, que determinan la disponibilidad de recursos hídricos en un determinado territorio, también resulta de notable interés estudiar la evolución de avenidas debido a sus drásticas consecuencias sobre las infraestructuras y poblaciones ribereñas. La figura 11 representa la evolución de los caudales medios diarios máximos anuales $\left(Q_{c}\right)$ y de los máximos instantáneos anuales (Qci) durante el período de estudio. Ambas variables están correlacionadas y no muestran ningún tipo de tendencia positiva o negativa en el tiempo. Los valores medios de Qcy Qci son de 49 y 89 $\mathrm{m}^{3} / \mathrm{s}$ respectivamente, $y$ en contadas ocasiones superan el valor doble del promedio.
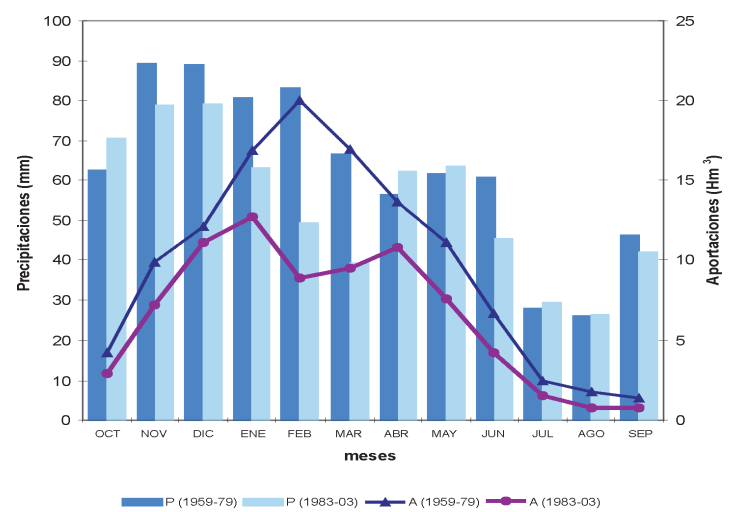

Figura 10: Comparación entre la distribución mensual de las aportaciones $(A)$ y precipitaciones $(P)$ en el curso alto del río Duero en los períodos comprendidos entre 1959-60/1979-80 y 1983-84/2003-04.

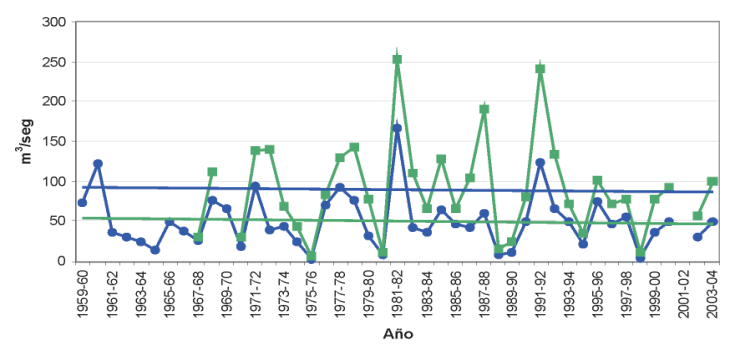

$\rightarrow-Q c-Q-Q c i$ - Lineal $(Q c)$ - Lineal (Qci)

Figura 11: Evolución interanual de los caudales medios diarios máximos anuales $\left(Q_{c}\right)$ y caudales máximos instantáneos anuales (Qci) en la estación de aforos de Molinos de Duero, 1959-60/2003-04. 
Respecto a las crecidas, de acuerdo con los criterios expuestos en el apartado 3, consideramos crecida moderada $(Q \times 3)$, avenida moderada $(Q \times 5)$ y avenida de baja frecuencia y alta intensidad $(Q \times 10)$ cuando el caudal medio diario supera $9,1 \mathrm{~m}^{3} \mathrm{~s}^{-1}, 15,2 \mathrm{~m}^{3} \mathrm{~s}^{-1} \mathrm{y}$ $30,3 \mathrm{~m}^{3} \mathrm{~s}^{-1}$ respectivamente (figura 12). En todos los casos, la tendencia temporal de las tres clases determinadas es negativa, siendo significativa en el caso de las crecidas moderadas $(Q \times 3)$, con un $R s=-0,41$; significativo al $99 \%$, en concordancia con la disminución de las aportaciones anuales señalada anteriormente. Si las avenidas decrecen es lógico pensar que el número de días en los que el río presente un estado de aguas bajas haya aumentado en el tiempo. Para ello, basándonos en el trabajo de MACHín et al. (2005), hemos cuantificado el número de días en el que el caudal del río es inferior al caudal diario medio anual dividido por tres y analizado cuál ha sido la tendencia de esta variable. De acuerdo con ello, durante el período 1959 y 2003 el $41,8 \%$ de los días no han superado el umbral de $1 \mathrm{~m}^{3} \mathrm{~s}^{-1}$ y el número de días al año con aguas bajas ha crecido de forma significativa $(R s=0,31$; valor-p $=0,041)$, pasando de menos de 130 al inicio del período a más de 180 a finales del mismo (figura 13).

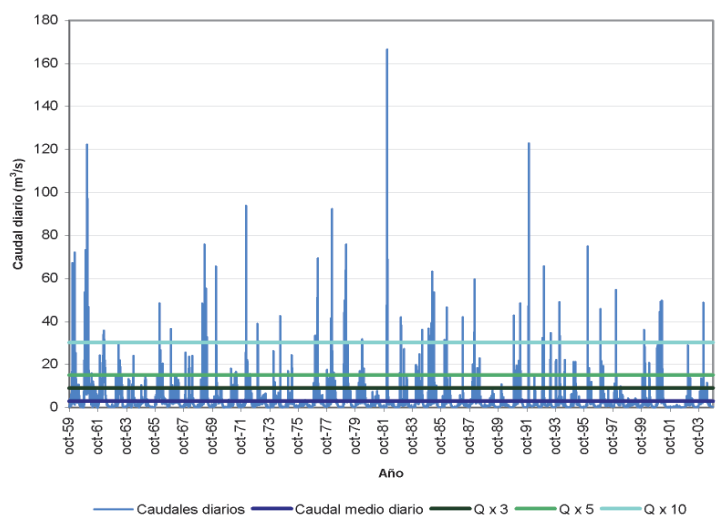

Figura 12: Evolución interanual de los caudales diarios y umbrales correspondientes a 3,5 y 10 veces el caudal medio anual en la estación de aforos de Molinos de Duero, 1959-60/2003-04.

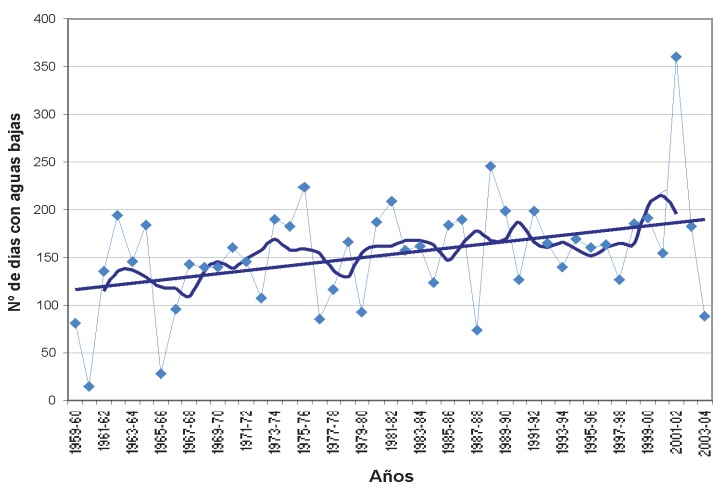

Figura 13: Evolución interanual del número de días anuales con régimen de aguas bajas o por debajo de la tercera parte del caudal medio anual en la estación de aforos de Molinos de Duero, 1959-60/2003-04.

\subsection{Cambios en el bosque}

A mediados del siglo $X X$, el bosque ocupaba una extensión de $98,3 \mathrm{~km}^{2}$ (equivalente al $72,8 \%$ de la superficie de la cuenca) de los cuales $19,3 \mathrm{~km}^{2}$ correspondían a formaciones jóvenes y de baja densidad de arbolado (Pinus sylvestris). El límite altitudinal del bosque se encontraba a $1.820 \mathrm{~m}$, a partir del cual tan sólo se desarrollaban formaciones arbustivas y pastizales de montaña. A comienzos del siglo XXI, el bosque había crecido ligeramente en superficie y en densidad: La superficie cubierta de bosque había ascendido a $104,9 \mathrm{~km}^{2}(77,7 \%$ del área de la cuenca) y el límite forestal alcanzaba la isohipsa de los $1.900 \mathrm{~m}$ (figura 14).

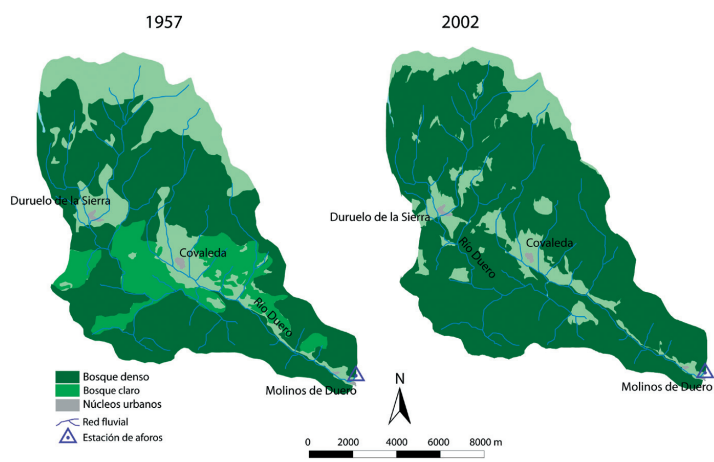

Figura 14: Distribución de la superficie forestal en 1957 y 2002 en la cabecera fluvial del río Duero.

\subsection{Interacciones entre las variables hidroclimáticas y el posible efecto del bosque}

La figura 15 muestra la evolución durante el período de estudio de las temperaturas, precipitaciones y aportaciones anuales, una vez normalizadas con la aplicación del índice $F$, destacando el acusado paralelismo entre precipitaciones y aportaciones. El coeficiente de correlación entre ambas variables es marcadamente significativo $(R=0,72$; valor-p < 0,001 ), y el grado de explicación de la variable dependiente (aportaciones) no aumenta de manera apreciable si introducimos en un análisis de regresión múltiple variables como la temperatura o el tiempo ( $R$ $=0,78$; valor $-\mathrm{p}<0,001$ ).

La siguiente variable de carácter dinámico que puede explicar la respuesta hidrológica de la cuenca son los cambios operados en su cobertura forestal. En este sentido, la figura 15 muestra que la relación entre precipitaciones y aportaciones no ha variado a lo largo del tiempo, ya que no existe un intervalo en el cual de forma sistemática la línea de las aportaciones haya estado por encima o por debajo de la correspondiente a las precipitaciones, acorde con la 
ausencia de cambios significativos habidos en la superficie del bosque (apartado 4.3.). Esta apreciación es confirmada además por el hecho de que no exista ninguna tendencia significativa en la correlación entre los residuales producto de la correlación entre precipitación y aportación año a año y el tiempo (Rs = -0,28; valor-p=0,059).

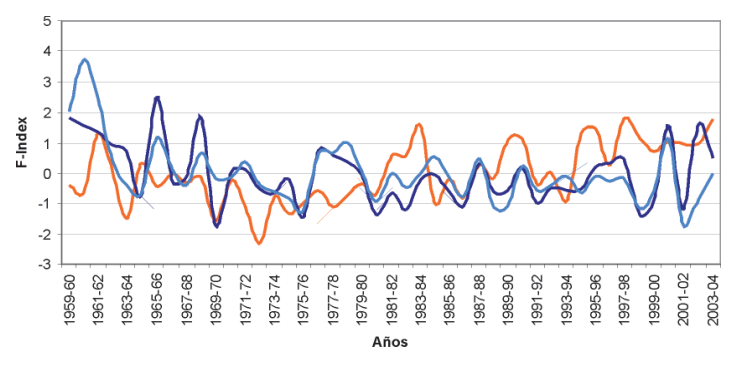

- $\mathrm{T}$ - $\mathrm{P}$ -

Figura 15: Evolución temporal de las temperaturas, precipitaciones y aportaciones normalizadas (1959-60/2003-04).

\section{Discusión y Conclusiones}

Los resultados del análisis de la evolución de las variables climáticas consideradas en este trabajo (temperaturas medias y precipitaciones) se adecúan a las tendencias observadas y publicadas por varios estudios realizados a distintas escalas, pero con algunos matices respecto a la magnitud $y$ estacionalidad de los cambios.

Con independencia de la escala de análisis (planetaria, europea, nacional o regional), todos los estudios de anomalías térmicas observadas durante el siglo XX y comienzos del XXI coinciden en describir un período de estabilidad e incluso descenso de las temperaturas entre mediados de los 40 y comienzos de los 70 del siglo pasado, para posteriormente iniciar un ascenso claro, con el registro de los años más cálidos a finales de los 90 y comienzos del siglo XXI (Jones et al., 2006; KLeIN TANK et al., 2002; BRUNET et al., 2007; Morales et al., 2005). En este sentido, la serie analizada ha mostrado un incremento significativo de las temperaturas durante el período estudiado, siendo especialmente llamativo a partir de 1972 con un ritmo de $+0,53^{\circ} \mathrm{C}$ por década. Este incremento es notablemente superior al observado por JONES et al. (2006) para el mismo período $\left(+0,17^{\circ} \mathrm{C}\right)$ a escala mundial y muy parejo al detectado por De CASTRO et al. (2005) y Morales et al. (2005) para España $\left(+0,58^{\circ} \mathrm{C}\right)$ y Castilla y León $\left(+0,49^{\circ} \mathrm{C}\right)$ respectivamente. Si a nivel mundial el calentamiento ha sido más evidente en los meses invernales, en el área estudiada los meses primaverales y estivales han sido los que han experimentando los mayores incrementos, coincidiendo con los análisis de BRUNET et al. (2007) y MoRALES et al. (2005) a escalas nacional y regional. El comportamiento de las temperaturas observado en la cabecera del río Duero en la provincia de Soria es similar al detectado en un estudio realizado en la cabecera del río Tormes, afluente directo del río en el sector más suroccidental de la cuenca en territorio español (CEBALLOS \& MORÁN, 2006), y se ajusta al patrón regional descrito por MORALES et al. (2005) y CEBALlos et al. (en prensa).

La consustancial variabilidad espacio-temporal de las precipitaciones no hace posible el diseño de patrones de comportamiento como en el caso de las temperaturas. En el territorio español, y nuestros resultados no son una excepción, una serie de trabajos desarrollados a distintas escalas concluyen mayoritariamente que las precipitaciones anuales no presentan ninguna tendencia estadísticamente significativa y en caso de haberla suele ser negativa. El decrecimiento marcado de las precipitaciones en los meses de febrero y marzo coincide con el observado por PAREDES et al. (2006) para el conjunto de la Península Ibérica o con los análisis de Ceballos \& MORÁN (2006) y NUNES (2007) realizados en otras cuencas incluidas en la red hidrográfica del Duero. Integrando la dinámica observada en las dos principales variables climáticas estudiadas, podemos concluir que la disminución de la lluvia en los meses invernales más el aumento de las temperaturas primaverales y estivales con toda probabilidad esté repercutiendo en una mayor capacidad del bosque para fijar y consumir agua, debido a la prevalecencia de los procesos de infiltración y evapotranspiración sobre los de escorrentía.

La evolución interanual de las aportaciones medidas en la estación de aforos de Molinos de Duero muestra un descenso apreciable de las mismas significativo al $99 \%$, coincidiendo con la tendencia registrada en otras cuencas comparables de la misma red hidrográfica: por ejemplo, río Coa (NUNES, 2007) en Portugal, río Arlanza en España (MACHíN et al, 2005) o el propio sistema fluvial integrado por los ríos Tormes, Huebra y Águeda también en España (Ceballos et al, 2008). El ajuste lineal de la serie estudiada apunta que en la actualidad la producción de agua de la cabecera del río Duero es un $59 \%$ menor que la registrada en los años iniciales de medición. Este significativo descenso desde un punto de vista cuantitativo coincide con el máximo de la horquilla establecida por GALLART y LLORENS (2003), quienes apuntan que las aportaciones de los principales ríos españoles han decrecido entre un 37 y un $59 \%$ en los últimos $50-60$ años. Si lo contrastamos con datos de cuencas de tamaño medio pertenecientes 
a la red hidrográfica del Duero es comparable al observado en el sistema de cuencas suroccidentales (el denominado sector E por la propia Confederación Hidrográfica del Duero), en donde el descenso de las aportaciones ha oscilado entre un 32 y un $66 \%$ (CEballos et al., 2008) e inferior al $80 \%$ estimado en el río Arlanza por MACHín et al. (2005).

Los resultados del análisis estadístico de evolución temporal de las series mensuales de aportaciones apuntan un descenso marcado entre febrero y septiembre, siendo los meses más críticos marzo, junio, julio, agosto y septiembre. Este resultado coincide con otros previamente publicados correspondientes a cuencas como la propia del Duero o el Ebro. Beguería et al (2003), en el caso del Ebro, señalan un descenso de las aportaciones marcado en los meses primaverales y estivales, destacando el caso de marzo. AdélIA Nunes (2007) en su análisis de la cuenca portuguesa del río Coa (cuenca del Duero) señala que los meses de febrero, marzo y abril fueron los que experimentaron las mayores reducciones, y la misma conclusión encontramos en los análisis de las cuencas del Arlanza (MACHín et al., 2005) y suroccidentales del Duero (CEBALLos et al., 2008). El descenso de las aportaciones registradas, sobre todo en los meses primaverales, ha tenido una importante repercusión: por un lado, en la producción anual de agua en la cuenca debido a que son meses con coeficientes mensuales de caudales superiores a 1 ( 1 representa la media anual) y, por otro, en la modificación detectada del régimen fluvial. Este hecho queda claramente reflejado en la figura 10 en donde es perceptible la transición de un régimen con un máximo de carácter pluvial a finales del invierno a un régimen bimodal.

Las aportaciones de la cuenca estudiada son de gran importancia para abastecer al primer gran embalse construido en el cauce del río Duero. La presa de la Cuerda del Pozo fue construida en 1941 con una capacidad para embalsar $249 \mathrm{Hm}^{3}$. Dicho embalse tiene actualmente diversas funciones que podemos resumir en producción de energía eléctrica, abastecimiento de agua potable, abastecimiento de agua de riego, regulación hídrica y actividades de recreo. El decrecimiento de los caudales y el cambio de régimen fluvial, observados en nuestro análisis, deberían ser considerados en la gestión del embalse, según ha sido estudiado en otras zonas como el Pirineo Aragonés (López Moreno et al., 2004). El carácter bimodal del régimen plantea la duda de si rellenar el embalse con las aportaciones estrictamente invernales, asegurando la disponibilidad de agua durante los meses más críticos pero perdiendo su capacidad de regulación ante eventuales crecidas en primavera; o bien dejar un margen de regulación, dependiendo la recuperación total del agua embalsada de las aportaciones primaverales, que en el caso de una primavera seca puede hipotecar la reserva de agua para riego o producción eléctrica. También es probable que este decrecimiento de las aportaciones magnifique el efecto del propio embalse sobre los caudales medidos aguas debajo de la presa, ya que se traduce en un incremento del denominado Impoundment Runoff (IR) index (o índice de retención de escorrentía) resultado de dividir la capacidad total del embalse por las aportaciones del conjunto de cuencas vertientes al mismo (BATALLA et al, 2004). Este cambio observado en el régimen fluvial en la cabecera del río Duero, pasando de un único pico de carácter pluvial a dos, se añade a la lista de cambios detectados en otros ríos de la misma cuenca en donde en algunos casos las cabeceras han perdido su carácter nival y en otros el estiaje se ha adelantado a la primavera temprana (CEBAllos et al., 2008).

Los cambios del bosque ocurridos en la zona de estudio en los últimos años no han sido significativos desde un punto de vista cuantitativo con un aumento superficial de sólo un $5 \%$. No obstante, sí hemos detectado un cierto cambio cualitativo en la configuración del bosque, con un incremento de la superficie de bosque maduro y por tanto del volumen de biomasa vegetal. A pesar de ello, el análisis de la evolución temporal de temperaturas, precipitaciones y aportaciones normalizadas (figura 15) no manifiesta una señal del bosque clara, como por ejemplo sí concluyen Beguería et al. (2003) en su análisis del Pirineo Aragonés, debido a que la línea correspondiente a las aportaciones no muestra sistemáticamente valores inferiores a la de las precipitaciones a partir de un determinado momento. Igualmente, la falta de relación entre el tiempo y los residuos de la correlación entre precipitaciones y aportaciones confirma la ausencia de una señal perceptible del bosque. No obstante hay dos hechos que quisiéramos subrayar y que sugieren una posible influencia del bosque en las aportaciones de la cuenca estudiada que probablemente sea perceptible a medio plazo: i) Los análisis de las tendencias temporales de las precipitaciones y aportaciones anuales (figuras 4 y 7 ) apuntan que el descenso de las aportaciones es más significativo que el de las precipitaciones. ii) Los análisis de las tendencias temporales de las series mensuales de ambas variables (figuras 5 y 8 ) no muestran una correspondencia clara entre ambas, ya que en el caso de las aportaciones el descenso es significativo entre los meses de febrero y octubre, mientras que en el caso de las precipitaciones 
sólo febrero registra un descenso significativo y meses como abril, mayo y agosto reflejan una tendencia positiva aunque no significativa. Estas diferencias en los valores mensuales entre dos variables que sí muestran una muy buena correlación entre sus series de datos anuales es posible que se deba a un aumento del consumo de agua del bosque en relación con el aumento de las temperaturas. La especie forestal predominante en la cuenca estudiada es el Pinus sylvestris, adaptada a inviernos fríos y veranos frescos (temperatura media del mes más cálido inferior a $20^{\circ} \mathrm{C}$ ). Si observamos la figura 3 , en las tres últimas décadas el aumento de las temperaturas ha sido muy significativo entre los meses de marzo y agosto lo que ha supuesto que se adelante de manera brusca el inicio del período de actividad vegetal al mes de marzo y que actualmente se supere el umbral de los $20^{\circ} \mathrm{C}$ en los meses de julio y agosto. En este nuevo escenario bioclimático, es lógico pensar que el consumo de agua por parte de los pinares de P. sylvestris esté aumentando de forma notable.

No obstante, de momento los resultados obtenidos en este trabajo corroboran los publicados en análisis previos que detectan una reducción de las aportaciones atribuible en gran medida a un decrecimiento de las precipitaciones en el tiempo (el análisis de correlación entre precipitaciones y aportaciones es de $R=0,72$; valor-p $<0,001$ ), y no a cambios en la cubierta vegetal; $y$ además parecen confirmar las conclusiones de TRIGO et al. (2004) cuando afirman que a escala de la Península Ibérica, el decrecimiento de las aportaciones en las grandes cuencas hidrográficas que desembocan en el Atlántico (Duero, Tajo y Guadiana) se atribuye a la disminución de las precipitaciones en los meses de invernales asociadas a fases negativas del índice NAO (North Atlantic Oscillation) durante los meses previos.

Otra evidencia de la dependencia de la respuesta hidrológica de la cuenca de la evolución de las precipitaciones, y de que las señales de la influencia del bosque sobre la producción de escorrentía superficial no son perceptibles de forma nítida en la actualidad, la tenemos en el comportamiento observado en los caudales medios diarios máximos anuales (Qc), los máximos instantáneos anuales (Qci) y en los distintos tipos de crecidas $(Q \times 3, Q \times 5$ y $Q \times 10)$. La ausencia de correlación significativa entre el tiempo y las variables $Q_{c}, Q_{c i}, Q \times 5$ y $Q \times 10$ muestra que el leve crecimiento del bosque detectado no ha sido suficiente para incrementar la capacidad reguladora de la cuenca sobre las crecidas de baja frecuencia y alta intensidad. Nuestros resultados no coinciden con los publicados, en referencia a los afluentes pirenaicos del Ebro, por LóPEZ MORENO et al. (2006) que sí han observado una tendencia temporal negativa en la magnitud de las crecidas que atribuyen al avance de la cobertura forestal tras el abandono de muchos campos en la montaña pirenaica, ya que no han detectado cambios en la frecuencia e intensidad de eventos pluviométricos extremos. Nosotros sólo hemos detectado una relación negativa estadísticamente significativa entre el tiempo y los días de crecida moderada $(Q \times 3)$, con un consecuente aumento del número de días con aguas bajas, que atribuimos al descenso de lluvias en los meses invernales que hipoteca la recarga hídrica del suelo y que necesariamente afecta al flujo base del río.

El patrón observado en Molinos de Duero, que podríamos resumir en que existe una muy buena correlación entre precipitaciones y aportaciones y que la superficie forestal cambia poco en varias décadas y por tanto no repercute de manera clara en la evolución de las aportaciones, es comparable al observado en estudios realizados, a distintas escalas, en otras cabeceras fluviales y redes hidrográficas dentro de la misma cuenca del Duero. En la cabecera del río Tormes $\left(83 \mathrm{~km}^{2}\right)$, Ceballos \& Morán (2006) apuntan una correlación altamente significativa entre precipitaciones y aportaciones $\left(r^{2}=0,71\right.$; valor-p < 0,001 ), no encontrando una señal evidente del tímido avance experimentado por el bosque (en 1957 ocupaba el $12 \%$ de la cuenca y en 2001 el 15,2\%). En la cabecera del río Arlanza $\left(349 \mathrm{~km}^{2}\right)$, MACHín ef al. (2005) también hallan un buen ajuste entre precipitaciones y aportaciones $\left(r^{2}=0,52\right.$; valor-p < 0,001 ), no demostrando el efecto de la señal del bosque para explicar el descenso de los caudales. En una cuenca de mayor tamaño, como es el caso del río Coa en Portugal con $2521 \mathrm{~km}^{2}$ de superficie, el trabajo de NUNES (2007) muestra una muy buena correlación entre precipitaciones y aportaciones $\left(r^{2}\right.$ $=0,82$; valor-p $<0,001$ ), pero no demuestra el posible efecto del tímido avance del bosque sobre la evolución de las aportaciones. Finalmente, el análisis integrado de las cuencas de los ríos Águeda, Huebra y Tormes por parte de Ceballos et al. (2008), ocupando una superficie superior a los $13.000 \mathrm{~km}^{2}$, muestra de nuevo que el crecimiento del bosque ha sido muy tímido, siendo los cambios cualitativos más importantes que los cuantitativos, y que la tendencia decreciente de las aportaciones de los ríos está muy correlacionada con la experimentada por las propias precipitaciones $\left(r^{2}=0,72\right.$; valor-p $\left.<0,001\right)$.

Estas conclusiones difieren significativamente respecto a los resultados publicados en referencia a otros 
territorios de la Península Ibérica. Por ejemplo, en el Pre-Pirineo Catalán (PoyAtos et al., 2003) detectan cambios sustanciales en la cobertura del bosque en las cuencas analizadas que pasa de un $39 \%$ en 1957 a un $64 \%$ en 1996, con un notable efecto de este hecho sobre la producción de agua en la cuenca debido a una mayor demanda evapotranspirativa, aumento de la interceptación e incremento de la discontinuidad espacial de la escorrentía superficial al haber más obstáculos en forma de manchas de vegetación (LLoRENs et al., 2005). BEGUería et al. (2003), en el Pirineo Aragonés, señalan que el descenso de los caudales de los ríos estudiados coincide con un cambio importante en el uso del suelo, relacionando la colonización por parte del bosque del $22 \%$ correspondiente de las tierras cultivadas de las cuencas con una reducción del $30 \%$ de las aportaciones a lo largo de la segunda mitad del siglo XX.

Por lo tanto, un aspecto clave, que debe ser resuelto en futuros trabajos con el estudio de nuevos casos, es conocer de manera más detallada a partir de qué umbral de cobertura vegetal comienza a ser clara y evidente la señal del avance del bosque sobre la cantidad de agua que circula superficialmente y la dependencia de esta interacción de la escala del análisis (tamaño de la cuenca y/o valores anuales o mensuales). Además, con independencia del crecimiento del bosque, deberán ser considerados los cambios fisiológicos de la vegetación, con un aumento de la transpiración, como respuesta al aumento del estrés hídrico debido al incremento de las temperaturas y descenso de las precipitaciones, y sus consecuencias sobre la producción de agua en las cuencas fluviales de cabecera.

\section{Agradecimientos}

Esta investigación ha sido posible gracias al apoyo financiero de la Junta de Castilla y León (Proyecto SA099A06).

\section{Bibliografía}

Batalla, R.; Gómez, C. M. \& Kondolf, M.G. (2004) "Reservoir-induced hydrological changes in the Ebro river basin (NE Spain)". Journal of Hydrology, 290: 117-136.

Beguería, S., López-Moreno, J. I., Lorente, A., Seeger, M., García-Ruiz, J. M. (2003) - "Assessing the effect of climate oscillations and land-use changes on streamflow in the Central Spanish Pyrenees". Ambio 32(4), 283-286.

Brown, A.E., Zhang, L., McMahon, T.A., Western, A.W., VERTESSY, R.A. (2005) - "A review of paired catchments studies for determining changes in water yield resulting from alterations in vegetation". Journal of Hydrology $310,28-61$.

Brunet, M.; Jones, P.D.; Sigró, J. ; Saladié, O. ; Aguilar, E. ; Moberg, A. Della-Marta, P.M.; Lister, D.; Walter, A. Y LóPEZ, D. (2007) - "Temporal and spatial temperature variability and change over Spain during 1850-2005". Journal of Geophysical Research, 112, D12117.

Ceballos, A. \& Morán Tejeda, E. (2006) - "Evolución de las aportaciones hídricas en una cuenca de montaña del Sistema Central: cabecera fluvial del río Tormes (1941-2004)". Cuadernos de Investigación Geográfica, 32: 7-28.

Ceballos, A.; Morán-Tejeda, E.; Luengo-Ugidos, M.A. \& LLORENTE-PINTO, J.M. (2008) - "Water resources and environmental change in a Mediterranean environment: the south-west sector of the Duero river basin (Spain)". Journal of Hydrology, 35 1: 126-138.

Ceballos, A., Morán Tejeda, E. \& Quirós Hernández, M. (en revisión) - "Evolución de las temperaturas y precipitaciones en las capitales de Castilla y León en el período 1961-2006". Revista Polígonos.

De Castro, M., Martín-VIde, J., Alonso, S. (2005) - "El clima de España: pasado, presente y escenarios de clima para el siglo XXI", in Moreno Rodríguez, J.M. (Eds), Evaluación preliminar de los impactos en España por efecto del cambio climático. Ministerio de Medio Ambiente y Universidad de Castilla-La Mancha, pp. 1-64.

De luis, M., García-Cano, M.F., Cortina J., Raventós, J., González-Hidalgo, J.C., SÁnchez, J.R. (2001) "Climatic trends, disturbances and short-term vegetation dynamics in a Mediterranean shrubland". Forest Ecology and Management, 147, 25-37.

Font Tulot, I. (2000) - Climatología de España y Portugal. Ediciones de la Universidad de Salamanca, 422 pp.

Gallart, F. \& Llorens, P. (2003) - "Catchment management under environmental change: impact of land cover change on water resources. Water International, 28(3): 334-340.

Gallart, F. \& LloRens, P. (2004) - Observations on land cover changes and water resources in the headwaters 
of the Ebro catchment, Iberian Peninsula". Physics and Chemistry of the Earth, 29: 769-773.

García-Ruiz, J.M.; Beguería Portugués, S.; López Moreno, J.I.; Lorente Grima, A. \& Seeger, M. (2001)Los recursos hídricos superficiales del Pirineo aragonés y su evolución reciente. Logroño, Geoforma Ediciomes, $192 \mathrm{pp}$.

IGME (1986) - Mapa Geológico 1:50.000. Hoja no 316, Quintanar de la Sierra. Centro de Publicaciones del Ministerio de Industria y Energía, Madrid.

IGME (1988) - Mapa Geológico 1:50.000. Hoja no 317, Vinuesa. Centro de Publicaciones del Ministerio de Industria y Energía, Madrid.

IPCC (2001) - Climatic Change 2001: The Scientific Bases. Contribution of Working Group I to the Third Assessment Report of the Intergovernmental Panel on Climate Change, in: Houghton, J.T., Ding, Y., Griggs, D.J., Noguer, M., van der Linden, P.J., Dai, X., Maskell, K., Johnson, C.A., (Eds), Cambridge University Press, United Kingdom, 881 pp.

JONES, P.D., D.E. PARKER, TJ. OSBORN, \& K.R. BRIFFA (2006)Global and hemispheric temperature anomalies-land and marine instrumental records. In Trends: $A$ Compendium of Data on Global Change. Carbon Dioxide Information Analysis Center, Oak Ridge National Laboratory, U.S. Department of Energy, Oak Ridge, Tenn., U.S.A. http://cdiac.ornl.gov/trends/ temp/ionescru/iones.html

KIELY, G. (1999) - "Climate change in Ireland from precipitation and streamflow observation". Advances in Water Resources, 23: 141-151.

KLEIN TANK, A.M.G. et al. (2002) - "Daily dataset of 20th-century surface air temperature and precipitation series for the European Climate Assessment". International Journal of Climatology, 22: 1441-1453.

Llorens, P.; Poyatos, R.; Rubio, C.; Latron, J. \& Gallart, F. (2005) - "El papel del bosque en los procesos hidrológicos. Ejemplos en las cuencas de investigación de Vallcebre (Prepirineo Catalán)". Cuadernos de Investigación Geográfica, 31 : 27-44.

López Moreno, J.I.; Beguería, S. \& García-Ruiz, J.M. (2004) - "The management of a large Mediterranean reservoir: storage regimens of the Yesa reservoir, upper Aragón river basin, Central Spanish Pyrenees". Environmental Management, 34 (4): 508-515.
López Moreno, J.I.; Beguería, S. \& García Ruiz, J.M. (2006) - "Trend in high flows in the central Spanish Pyrenees. Response to climatic factors or to land-use change?". Hydrological Sciences -Journal- des Sciences Hydrologiques, 51 (6): 1039-1050.

Machín, J.; Navas, A.; Doménech, S. \& López-VICENTE, $M$. (2005) - "El río Arlanza en cabecera: evolución reciente y tendencias en condiciones de cambio global". Cuadernos de Investigación Geográfica, 31: 77-95.

MIMAM (2000) - Libro Blanco del Agua en España. Ministerio de Medio Ambiente, Madrid. 637 pp.

Morales, C.; Ortega, M.T.; labajo, J.l. \& Piorno, A. (2005) - "Recent trends and temporal behavior of thermal variables in the region of Castilla-León (Spain)". Atmósfera, 18(2): 71-90.

Nunes, A. (2007) - "Recursos hídricos na bacia do rio Côa: relações com a variabilidade climática e mudanças no uso do solo". Iberografias, 10: Territorios e Culturas Ibéricas II, 71-86.

OLIVER, J.F. (1980) - "Monthly precipitation distribution: a comparative index". Professional Geogr. 32, 300-309.

Paredes, D.; Trigo, R.M., García-Herrera, R. y Franco TRIGO, I. (2006) - "Understanding Precipitation Changes in Iberia in Early Spring: Weather Typing and StormTracking Approaches". Journal of Hydrometeorology, 7(1): 101-113.

Poyatos, R.; LAtron, J. \& Llorens, P. (2003) - "Land-use and land cover change after agricultural abandonment. The case of a Mediterranean mountain area (Catalan Pre-Pyrenees)". Mountain Research and Development, 23 (4): 52-58.

Ruiz de la ToRre, J. (1995) - Mapa Forestal de España, 1:200.000. Soria, hoja 6-4. Ministerio de Agricultura, Pesca y Alimentación.

SCIAN, B., Donnarl, M. (1997)- "Retrospective analysis of the palmer Drought Severity Index in the semi-arid pampas region, Argentina". International Journal of Climatology 17, 313-322.

Shorthouse, C., Arnell, N. (1999) - "The effects of climate variability on spatial characteristics of European river flows". Physics and Chemistry Earth 24(1-2), 7-13.

SNEYERS, R. (1992) - "Use and misuse of statistical methods for detection of climatic change". Climate Change Detection Project, Report on the Informal 
Planning Meeting on Statistical Procedures for Climate Change Detection, WCDMP, (20), J76-J81.

Trigo, R. M.; Pozo-VÁzquez, D.; Osborn, T.J.; Castro Díez, Y.; Gámiz-Fortis, S. \& Esteban-ParRa, M.J. (2004) "North Atlantic Oscillation influence on precipitation, river flow and water resources in the lberian Peninsula". International Journal of Climatology, 24: 925-944.

Verburg, P.H.; SChUIP, CJ.S.; Witte, N. \& VeldKamp, A. (2006)"Dowscaling of land use change scenarios to asses the dynamics of European landscapes". Agriculture, Ecosystems and Environment, 114 (1): 39-56.

Yue, S., Pilon, P., Cavadias, G. (2002) - "Power of the Mann-Kendall and Spearman's rho tests for detecting monotonic trends in hydrological series". Journal of Hydrology 259, 254-271. 